Slavica

bruxellensia

\section{Slavica bruxellensia}

Revue polyphonique de littérature, culture et histoire

slaves

\section{$11 \mid 2015$}

Littérature et philosophie

\title{
La fascination des lecteurs pour la mort en parade
}

Sur le livre Jak uwieść czytelnika? Jonathan Carroll i Andrzej Sapkowski wobec kulturowych koncepcji śmierci de Agnieszka Moroz

\section{Anna Jaworska}

Traducteur : Katia Vandenborre

\section{OpenEdition}

\section{Journals}

Édition électronique

URL : http://journals.openedition.org/slavica/1704

DOI : 10.4000/slavica. 1704

ISSN : 2034-6395

Éditeur

Université libre de Bruxelles - ULB

\section{Référence électronique}

Anna Jaworska, «La fascination des lecteurs pour la mort en parade », Slavica bruxellensia [En ligne],

11 | 2015, mis en ligne le 15 février 2015, consulté le 22 septembre 2020. URL : http://

journals.openedition.org/slavica/1704; DOI : https://doi.org/10.4000/slavica.1704

Ce document a été généré automatiquement le 22 septembre 2020.

\section{(†) $\ominus$}

Les contenus de Slavica bruxellensia sont mis à disposition selon les termes de la Licence Creative Commons Attribution - Pas d'Utilisation Commerciale - Pas de Modification 3.0 France. 


\section{La fascination des lecteurs pour la mort en parade}

Sur le livre Jak uwieść czytelnika? Jonathan Carroll i Andrzej Sapkowski wobec kulturowych koncepcji śmierci de Agnieszka Moroz

Anna Jaworska

Traduction : Katia Vandenborre

\section{RÉFÉRENCE}

Moroz A., Jak uwieść czytelnika? Jonathan Carroll i Andrzej Sapkowski wobec kulturowych koncepcji śmierci, Pisarze.pl, Varsovie, 2014, 92 p. 
1 Publié en 2014 par la maison d'édition Pisarze.pl, Jak uwieść czytelnika? Jonathan Carroll $i$ Andrzej Sapkowski wobec kulturowych koncepcji śmierci (Comment séduire le lecteur? Jonathan Carrol et Andrzej Sapkowski par rapport aux conceptions culturelles de la mort) est le premier ouvrage scientifique de la poétesse Agnieszka Moroz qui est actuellement doctorante en philologie polonaise à l'Université de Szczecin. L'auteure a décidé de présenter le résultat de ses recherches sur les conceptions culturelles de la mort dans des œuvres choisies d'Andrzej Sapkowski et Jonathan Carroll à un large éventail de lecteurs. Quoique surprenante à première vue, la juxtaposition de ces deux écrivains issus de cultures différentes est loin d'être

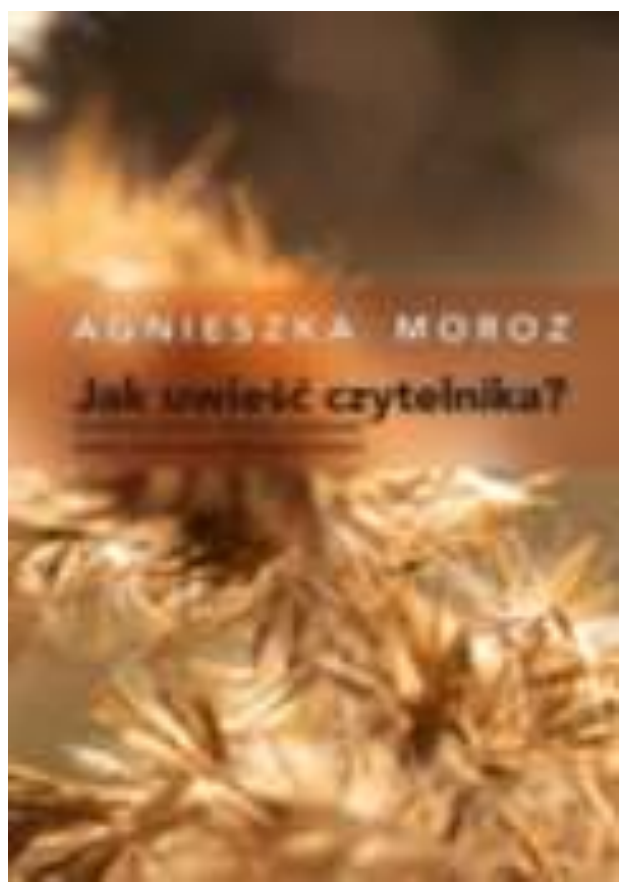
infondée. Moroz démontre, en effet, de manière convaincante que ces écrivains ont de nombreux points communs.

2 Lauréat de prestigieux prix littéraires, Sapkowski (né en 1948) est l'auteur polonais de fantasy le plus traduit après Stanisław Lem. Sa saga en cinq tomes sur Geralt de Riv a connu un grand succès. Le fait qu'elle ait été remise accompagnée d'un autographe de l'auteur et du jeu vidéo Wiedźmin 2 (Le Sorceleur 2) au président Barack Obama, lors de la visite de celui-ci en Pologne en 2011, témoigne de l'extraordinaire popularité dont elle jouit parmi les Polonais. L'œuvre de Sapkowski est dès lors devenue une source de fierté nationale.

3 Jonathan Carroll (né en 1949) est, quant à lui, un écrivain américain. Il peut sembler étonnant qu'il n'ait jamais été particulièrement reconnu dans son pays. Les éditeurs américains demeurent réticents à publier ses romans, notamment à cause de la multiplicité générique de ses livres. Lui-même les compare à des salades mélangées sauf qu'à la place des tomates, concombres ou carottes, ils contiennent des éléments de fantastique, de psychologie et d'horreur avec une touche de magie. Ce mélange est néanmoins du goût des lecteurs polonais, si bien que certaines de ses œuvres ont paru exclusivement sur le marché polonais.

4 Ainsi, nous voyons que ces deux écrivains qui ont presque le même âge ont atteint des sommets de popularité en Pologne, tout en étant citoyens de deux pays différents. Nombre d'entre nous pourraient s'inquiéter de savoir ce qui a conduit à cette situation. Y sont-ils arrivés de la même manière ? Ont-ils appliqué les mêmes techniques ? Dans quelle mesure leurs romans sont-ils similaires ? En quoi diffèrent-ils ? Assouvissent-ils les désirs profondément cachés des lecteurs?

5 Le premier chapitre du livre de Moroz fournit déjà un élément de réponse à ces intrigantes questions, puisqu'il est consacré aux méthodes qui permettent de gagner la reconnaissance du lecteur. L'auteure remarque à juste titre qu'elles sont appliquées non seulement par Sapkowski et Carroll, mais aussi par J. K. Rowling et Stephen Meyer. La chercheuse appuie ses observations sur des affirmations publiées sur des forums et 
des portails Internet, rendant ainsi compte du rôle que jouent les goûts des destinataires dans la création des romans lus. Le chapitre ne manque pas non plus de réflexions sur la relation contemporaine au livre qui, dans le monde d'aujourd'hui, devient de plus en plus souvent un produit facile à digérer et à vendre, et non plus un festin exquis pour l'âme.

Ensuite, Moroz emmène son lecteur dans un voyage dans le temps et montre comment le rapport à la mort s'est modifié au travers des siècles. Dans ses considérations, elle donne des exemples issus notamment de la Bible, de la mythologie grecque, des croyances populaires, $\mathrm{du}$ folklore, des traditions celtique, anglo-saxonne et germanique. L'auteure mentionne par ailleurs de nombreuses œuvres plastiques, sans parler des quelques curiosités qui font varier le récit. L'une d'elle concerne la longévité moyenne des gens au début du XIV siècle.

7 Dans le dernier chapitre, la chercheuse analyse les manières dont la mort est représentée dans les œuvres de Carroll et Sapkowski. Selon elle, ils emplissent leurs romans de motifs étonnants et souvent sombres non seulement pour intriguer le destinataire ou rendre la réalité décrite plus extraordinaire, mais aussi pour répondre à l'intérêt suscité par la mort et amener les lecteurs dans des sphères inconnues de la métaphysique, de la magie ainsi que du rêve. À cet égard, les recherches perspicaces menées par la doctorante sur les modèles de certains personnages s'avèrent particulièrement précieuses.

8 La couverture du livre n'est pas non plus sans importance car elle correspond parfaitement à la thématique abordée. On y voit la photo d'une plante fanée dans des couleurs automnales avec différentes nuances d'orange et de brun, dont la symbolique de l'éphémère plonge d'ores et déjà le lecteur dans la rêverie. Elle nous rappelle aussi une vérité brutale : bien que de nombreux s'y soient essayés, nul n'est encore parvenu à échapper à la parade de la mort.

\section{AUTEURS}

\section{ANNA JAWORSKA}

Etudiante de Master à l'Université Libre de Bruxelles 